\title{
Segregación residencial de ingresos en el Gran Santiago, 1992-2002: una estimación robusta
}

Claudio A. Agostini. Universidad Adolfo Ibañez, Santiago, Chile. Daniel Hojman. Universidad de Chile, Santiago, Chile.

Alonso Román. Centro de Estudios de Conflicto y Cohesión Social, Santiago, Chile. Luis Valenzuela. Universidad Adolfo Ibañez, Santiago, Chile.

RESUMEN | El desarrollo económico de Chile en las últimas décadas ha impactado fuertemente el crecimiento de las ciudades y la forma en que las desigualdades sociales se reflejan espacialmente en ellas. Medir con precisión la segregación urbana es, así, relevante, pues además permite diseñar políticas públicas que reduzcan sus efectos negativos. Sin embargo, mediciones previas en Chile tienen limitaciones metodológicas que restringen su validez y comparabilidad. En el presente estudio se utiliza una nueva metodología que combina datos de encuestas con información censal, a fin estimar ingresos de hogares y, con ello, indicadores precisos y estadísticamente robustos de segregación residencial. Adicionalmente, se estima un indicador de segregación que captura de mejor forma el hecho de que los ingresos son una variable continua. Los resultados muestran que la segregación de los más pobres es mayor que la de los más ricos, que aumentó entre 1992 y 2002, y que existe, además, una heterogeneidad importante en la contribución de cada comuna a la segregación en Santiago.

PALABRAS CLAVE | áreas metropolitanas, segregación, distribución espacial.

ABSTRACT | Chile's economic development in recent decades has greatly impacted cities' development and the way social inequalities are spatially reproduced in them. Therefore, an accurate measurement of urban segregation is relevant and allows designing public policies to reduce their negative effects. However, previous measurements in Chile have methodological limitations that restrict their validity and comparability. The new methodology used combines survey data with census data to estimate household income and then statistically robust indicators of residential segregation. Additionally, it develops valuation for an indicator of segregation that best captures income as a continuous variable. The findings show that segregation of the poor is greater than that of the richest and that increased between 1992 and 2002. Municipality's contribution to Santiago's segregation has a significant heterogeneity.

KEY WORDS | metropolitan areas, segregation, spatial distribution.

Recibido el 2 de marzo de 2015, aprobado el 4 de diciembre de 2015

E-mail: C. Agostini, claudio.agostini@uai.cl| D. Hojman, dhojman@fen.uchile.cl|A. Román, roman.amarales@gmail.com | L. Valenzuela, luis.valenzuela@uai.cl 


\section{Introducción}

En las últimas décadas, Chile ha experimentado una transformación económica, política y social de gran envergadura. La urbanización, la evolución de las ciudades y de las interacciones al interior de estas son parte esencial de esa transformación. Este desarrollo y su impacto han resultado en una situación contradictoria. Por una parte, desde comienzos de los años noventa, el desarrollo económico y social se ha caracterizado por un crecimiento económico sostenido y políticas sociales activas que se han reflejado, por ejemplo, en que el ingreso de un hogar promedio se ha duplicado desde 1990, en una caída sustancial de la pobreza absoluta, ${ }^{1}$ y en un acceso creciente de la población a bienes y servicios básicos. Por otra parte, esta etapa de modernización capitalista parece estar asociada en Chile a desigualdades sociales sustantivas y a signos de una menor cohesión social en ciertas dimensiones. La alta desigualdad del ingreso se ha mantenido relativamente estable (el coeficiente de Gini ha fluctuado en un rango de entre 0,54 y 0,52 en los últimos 25 ańos), existe un acceso desigual de los distintos estratos socioeconómicos a la salud y la educación, la confianza interpersonal es la más baja de la ocDe y de América Latina, y la segregación socioeconómica en las escuelas es la más alta registrada a nivel mundial (Organisation for Economic Cooperation and Development [OECD], 2010).

La segregación urbana, entendida como la falta de interacción entre distintos grupos sociales, destaca entonces como una dimensión clave. Si bien la relación existente entre desigualdad y segregación residencial en Chile ha sido objeto de análisis y debate (Agostini, 2010; Ruiz-Tagle \& López, 2014; Sabatini \& Brain, 2008; Sabatini, Cáceres \& Cerda, 2001; Sanhueza \& Larrañaga, 2007; Watson, 2009), lo relevante es que esta segregación expresa y reproduce en el territorio las desigualdades y exclusiones sociales. ${ }^{2}$ Un punto de partida para analizar la relación entre desigualdad y segregación y para diseñar políticas públicas que mitiguen sus efectos negativos, es tener una medición confiable y replicable de la segregación residencial en las ciudades chilenas.

Este artículo introduce una nueva metodología para estimar con precisión los ingresos de los hogares y con ello medir la segregación por ingresos en el Gran Santiago, una capital que concentra nada menos que un $40 \%$ de la población nacional. La literatura previa sobre segregación residencial en el Gran Santiago ha señalado que el nivel de segregación sería elevado y comparable al de otras metrópolis latinoamericanas, aunque de menor grado que el de ciudades norteamericanas (Lambiri \& Vargas, 2011; Rodríguez \& Arriagada, 2004; Sabatini, 2006; Sabatini, Wormald, Sierralta \& Peters, 2010), y que su evolución ha sido desfavorable para la integración social en las últimas décadas (Sabatini et al., 2010; Sanhueza \& Larrañaga, 2007; Rodríguez, 2001). Sin embargo, la medición de la segregación residencial por características socioeconómicas en Chile ha tenido limitaciones metodológicas importantes que dificultan su correcta medición y, por ende, su comparación en el tiempo y con otras urbes. Estas limitaciones y posibles sesgos estadísticos en

Entre 1990 y 2003, la tasa de pobreza nacional cayó de 38,3\% a 20,1\%, y luego a 7,8\% en 2013 .

2 A ello se agregan los riesgos para la convivencia y la legitimidad democrática de una comunidad fragmentada. 
estudios previos, se deben en parte a la ausencia de variables socioeconómicas estables a nivel de hogares en el censo y a la poca representatividad a escalas geográficas más desagregadas que tienen las variables de ingreso en otras encuestas (la Encuesta de Caracterización Socioeconómica Nacional [Casen] o la Encuesta Origen Destino Santiago, por ejemplo). En tal sentido, las mediciones de segregación basadas en ingresos que se realizan en este trabajo son una contribución importante en esa dimensión.

Trabajos recientes destacan las posibles limitaciones de la literatura de segregación en Chile. Por ejemplo, Ruiz-Tagle y López (2014) y Opazo (2014) resumen una serie de aprensiones metodológicas relevantes, tanto económicas como sociológicas. En primer lugar, la caracterización de los grupos socioeconómicos se ha basado generalmente en categorías de marketing, usando directamente la información del censo sobre la tenencia en cada hogar de bienes durables y el nivel educacional del jefe de hogar. Un problema central de esta aproximación es que dichas categorías son inestables en el tiempo y no necesariamente capturan la estratificación y las desigualdades entre hogares. Esto es particularmente cierto si se lo aplica al período entre los censos de 1992 y 2002, porque el fuerte crecimiento económico y la significativa reducción de la pobreza produjeron cambios en los niveles y patrones de consumo, aumentando en forma importante el acceso a los bienes con que se define a los grupos socioeconómicos (Agostini, 2010). Algo similar, aunque más gradual en el tiempo, ocurrió con las diferencias basadas en niveles de educación, considerando que la cobertura escolar primaria y secundaria es hoy universal y el acceso a educación terciaria aumentó significativamente. Esto podría llevar a clasificar segmentos de ingreso medio como parte de la elite socioeconómica.

En segundo lugar, las medidas de segregación utilizadas hasta ahora -como el índice de disimilitud- se derivan directamente de aquellas utilizadas en la literatura sobre segregación racial y no son necesariamente apropiadas para capturar segregación por estratificación socioeconómica. Hay a lo menos dos razones para ello. Por una parte, estas medidas establecen una partición de la población en dos grupos. ${ }^{3}$ Además de la fragilidad asociada a la elección de cortes arbitrarios para definir dos grupos, no es claro que la heterogeneidad socioeconómica, la estratificación y las desigualdades subyacentes que captura la distribución del ingreso u otra variable de nivel socioeconómico puedan capturarse a partir de solo dos categorías. ${ }^{4}$ Por otra parte, a un nivel más conceptual, a diferencia de los grupos caracterizados a partir de raza o religión, los grupos definidos a partir de una variable socioeconómica están ordenados: un hogar de ingreso bajo está más cerca de uno de ingreso medio que de uno de altos ingresos.

Por último, no se ha establecido un método consistente para analizar la segregación en distintas escalas espaciales. Es decir, los estudios existentes no muestran una aproximación que permita descomponer la segregación de la ciudad según el aporte de subunidades como barrios o comunas.

3 En la literatura sobre segregación racial en Estados Unidos, lo central era capturar la separación entre blancos y negros.

4 Es distinta una sociedad donde las capas medias están integradas con los pobres y separadas de los hogares de ingreso alto, que otra en que individuos de las capas medias interactúan intensamente con los grupos de nivel socioeconómico alto, pero no con los de nivel socioeconómico bajo. 
En este artículo nos hacemos cargo de algunas de estas críticas, en particular respecto a la estimación de ingresos para construir índices de segregación y la obtención de errores estándar que permitan hacer comparaciones estadísticamente válidas en el tiempo. Con tal fin, se utiliza una nueva metodología para estimar ingresos a nivel de hogares y medir la segregación residencial con alta precisión estadística, incluso en zonas geográficas pequeñas. La metodología se basa en avances econométricos recientes para "espacializar" los ingresos, en los cuales se combinan encuestas de hogares, que no son representativas a niveles geográficos muy desagregados como distritos censales o manzanas-, con el censo, que sí lo es, pero no contiene información sobre ingresos. A diferencia de otros trabajos que extrapolan ingresos de hogares a partir de un modelo de regresión sin modelar los errores (Sanhueza \& Larrańaga, 2007), esta metodología permite recuperar desigualdades de ingreso local y estimar errores asociados a las medidas de segregación, lo cual evita sesgos importantes en la materia. Adicionalmente, la estimación de errores permite establecer si los cambios en el tiempo son significativos y no simplemente ruido estadístico. Técnicas similares han sido utilizadas por Agostini y Brown (2007, 2010a, 2010b, 2011) para establecer medidas robustas de pobreza y desigualdad locales en Chile. A nuestro entender, el presente estudio es el primero en usar esta metodología para analizar la segregación residencial en el contexto latinoamericano.

Una segunda innovación metodológica respecto a la literatura anterior es el uso de medidas que capturan mejor la naturaleza continua de la variable de diferenciación socioeconómica entre los hogares; en este caso, los ingresos. Estudios previos usan el índice de disimilitud u otros índices tradicionales y aplicados a dos grupos. ${ }^{5}$ Nuestro trabajo usa este índice para cualquier división arbitraria de cortes de grupos por deciles, permitiendo comparaciones consistentes en el tiempo. Se introduce, además, un índice que captura la continuidad del ingreso: el Centile Gap Index (CGI) (Watson, 2009).

Los resultados obtenidos con la nueva metodología contribuyen a establecer los hechos estilizados de la segregación residencial en el Gran Santiago y su evolución entre 1992 y 2002, mediante la aplicación de una metodología de gran consistencia. En particular, usando índices tradicionales de segregación encontramos que la segregación de los más pobres es considerablemente mayor que la de los más ricos. Tanto en 1992 como en 2002, la segregación del 10\% de mayores ingresos presenta niveles del índice de disimilitud en torno a 0,53, es decir, más de un 53\% de este grupo debería cambiar su residencia a sectores menos homogéneos para lograr una integración perfecta en el espacio. Al mismo tiempo, si bien la segregación de los pobres en el Gran Santiago ostenta niveles comparables con los de áreas metropolitanas en Estados Unidos (Iceland \& Wilkes, 2006), la medida CGi es sustancialmente mayor, análoga solo a la de las áreas metropolitanas más segregadas de Norteamérica (Watson, 2009). Finalmente, y en contraste con Sabatini et al. (2010), nuestras estimaciones sugieren un alza estadísticamente significativa de la segregación residencial entre los años 1992 y 2002.

Sanhueza y Larrañaga (2007), por ejemplo, dividen la población entre un grupo vulnerable y el resto de la población. 
Exploramos también la heterogeneidad comunal de la contribución a la segregación de cada comuna. Al contrastar esta desagregación con mapas de localización tanto de pobreza como de altos ingresos, y datos de crecimiento poblacional por comuna, los resultados ilustran patrones interesantes de la dinámica urbana. La consolidación del sector oriente de Santiago como atractor de los hogares del decil más rico dentro de la ciudad, está asociada con una dinámica heterogénea en las comunas del "barrio alto". Hay aumentos de segregación en sectores nuevos y suburbanos, como Lo Barnechea, Peñalolén y parte de Huechuraba (como sugieren Ruiz-Tagle \& López, 2014) y, en contrapartida, una caída de la segregación en Providencia, La Reina, Vitacura y Las Condes. Se evidencia también un empobrecimiento relativo durante el decenio 1992-2002 de comunas céntricas como Santiago, San Miguel y Quinta Normal, lo cual se asocia con aumentos de segregación. Sin embargo, los cambios con mayor impacto en la segregación urbana provienen de comunas con un gran crecimiento poblacional, como Puente Alto, Quilicura y San Bernardo. Buena parte de ese crecimiento se asocia con guetos urbanos en condominios de vivienda social construidos durante la década de los noventa y la expansión de zonas de pobreza en la periferia.

\section{Metodología utilizada en la estimación de segregación}

La metodología general para estimar indicadores de segregación que tengan validez estadística a niveles desagregados utiliza datos de encuestas de hogares que incluyen información de ingresos, para imputar ingresos a datos censales que no contienen información de ingresos de los hogares (Hentschel, Lanjouw, Lanjouw \& Poggi, 1999). Luego, con los ingresos imputados en el censo a nivel de hogar, es posible estimar los indicadores de segregación a niveles más desagregados que los que estadísticamente permiten las encuestas de hogares. Esta metodología se implementa en dos etapas. En la primera, se estima un modelo de ingreso por hogar con los datos de las encuestas, pero considerando solo las variables explicativas comunes a la encuesta de hogares y el censo. En la segunda, se estima el nivel esperado de los indicadores de segregación, condicional a las características observables de la población en el censo.

Más formalmente, la metodología consiste en estimar la distribución conjunta del ingreso de los hogares y un vector de variables que covarían con el ingreso. Al restringir en la primera etapa el set de variables explicativas a aquellas características observables de personas y hogares que se encuentran también en el censo, la distribución conjunta estimada puede ser usada para generar la distribución de los ingresos para cualquier subconjunto de la población en el censo, condicional a las características observadas de ese subconjunto de la población. De esta forma, es posible obtener la distribución condicional, la estimación punto y el error estándar de los indicadores de segregación que dependen del ingreso de los hogares (Elbers, Lanjouw \& Lanjouw, 2003).

La validez de esta metodología descansa en un supuesto fundamental: implícitamente se asume que el modelo estimado usando los datos de la encuesta de hogares, es aplicable a las observaciones del censo. Si el año del censo y la encuesta coinciden 
o son cercanos en el tiempo, el supuesto no es muy restrictivo. Tal es el caso de los datos utilizados en este trabajo: la encuesta Casen del año 1992 junto al Censo 1992 y la encuesta Casen del año 2003 junto al Censo 2002.

\section{Metodología econométrica}

El detalle metodológico de las dos etapas de la estimación se describe formalmente a continuación. ${ }^{6}$ En la primera etapa, se estima un modelo que permite explicar el ingreso per cápita del hogar $h\left(Y_{h}\right)$, perteneciente a un grupo o clúster $c$ en la población, con un conjunto de características observables del hogar $\left(X_{h}\right)$. Estadísticamente, esto equivale a estimar la expectativa condicional del ingreso. Para ello se considera como forma funcional la proyección lineal de la expectativa condicional expresada en forma de error:

$$
\ln Y_{h c}=E\left[\ln Y_{h c} / X_{h c}\right]+u_{h c}=X_{h c} \beta+u_{h c}
$$

La variable dependiente en la estimación es el logaritmo natural del ingreso. Adicionalmente, se asume que el vector de errores $u$ tiene una distribución con media cero y matriz varianza-covarianza $\sum: f\left(0, \sum\right)$. Por último, para permitir algún grado de correlación entre los hogares que están en cada clúster, se considera la siguiente especificación para la estructura del error:

$$
u_{h c}=\eta_{c}+\mathcal{E}_{h c}
$$

donde $\boldsymbol{\eta}$ es el componente del error asociado al clúster $c$ y $\boldsymbol{\varepsilon}$ es un error idiosincrático. Se asume que estos dos componentes de la estructura del error son independientes entre sí y no están correlacionados con las variables observables $X_{h c}$.

En esta primera etapa, se estima el modelo (1) con la estructura (2) utilizando solo los datos de las encuestas de hogares. Como resultado de la estimación, se obtiene un conjunto de parámetros estimados del modelo consistentes en: el vector de coeficientes $\beta$, la matriz varianza-covarianza asociada a este vector y los parámetros que describen la distribución de los errores $\sum$.

En la segunda etapa, se combinan los parámetros estimados en la primera etapa con las características observables de cada individuo u hogar en el censo, para generar valores predichos del logaritmo del ingreso y los errores relevantes. Para estos efectos se utiliza un bootstrap que simula los valores del ingreso de cada hogar. Los valores simulados se basan en la predicción de los ingresos y los términos de error $\eta$ y $\boldsymbol{\varepsilon}$.

$$
\hat{Y}_{h c}=\exp \left(X_{h c} \hat{\beta}+\eta_{c}+\hat{\varepsilon}_{h c}\right)
$$

$6 \longdiv { \text { Un mayor detalle se encuentra en Agostini y Brown (2007, 2010a, 2010b) y Agostini, Brown y Góngora } }$ (2008), que utilizan la misma metodología para estimar desigualdad y pobreza comunal en Chile. 
donde, para cada hogar, los dos componentes del término de error son obtenidos de la distribución empírica descrita por los parámetros en la primera etapa. ${ }^{7}$ De igual forma, los coeficientes $\hat{\beta}$ se obtienen de la distribución descrita por los estimadores de $\beta$ en la primera etapa y la matriz varianza-covarianza asociada a ellos. ${ }^{8}$

Por último, para generar los indicadores de segregación, se utiliza el set completo de valores simulados de $\hat{Y}_{h c}$ para calcular el valor esperado de dichos indicadores para cada subgrupo o clúster de interés. El procedimiento se repite n veces, obteniendo cada vez un nuevo set de coeficientes $\beta$ y errores para cada simulación.? Luego, para cada subgrupo, se calcula la media y la desviación estándar del indicador de segregación sobre el total de simulaciones; ambas -la media y la desviación estándar- corresponden finalmente a las estimaciones punto del indicador y los errores estándar de estos estimadores, respectivamente.

De esta forma, lo que se realiza es implementar simulaciones de Montecarlo para calcular el valor esperado de cada índice de segregación $(S)$, condicional en la regresión estimada en la primera etapa; la varianza generada por el componente idiosincrático del ingreso per cápita de los hogares; y el vector de gradientes que determina la matriz varianza-covarianza. Con cada simulación se obtienen 100 vectores de términos de error de la distribución estimada en la primera etapa. Luego, con cada uno de estos vectores se calcula un valor para $S\left(\hat{S}_{d}^{E}\right.$ en la ecuación siguiente) y, finalmente, el valor esperado simulado para $S\left(\tilde{S}^{E}\right.$ en la ecuación siguiente) es la media para las 100 réplicas:

$$
\tilde{S}^{E}=\frac{1}{100} \sum_{d=1}^{100} \hat{S}_{d}^{E}
$$

De la misma manera, la varianza de $S$ es estimada simplemente usando la fórmula de la varianza con las 100 estimaciones punto del índice de segregación generadas con el bootstrap:

$$
\hat{V}_{\text {modelo }}=\frac{1}{100} \sum_{d=1}^{100}\left(\hat{S}_{d}^{E}-\tilde{S}^{E}\right)^{2}
$$

\section{Índices de segregación}

La metodología econométrica presentada puede utilizarse a fin de estimar indicadores de segregación para distintos subgrupos de la población -incluyendo, por ejemplo, grupos geográficamente desagregados-, a niveles de representatividad y precisión estadística que no es posible si se utilizaran solo los datos de encuestas

7 Para los dos componentes del error, hogar y clúster, se utiliza una distribución normal multivariada.

8 Utilizando el teorema del Límite Central, se asume que la distribución del vector $\beta$ converge a una distribución normal multivariada.

9 El criterio para definir el número n es que los resultados no cambien al aumentar las simulaciones. 
de hogares, como la Casen. Esto es particularmente cierto para las encuestas Casen 1992 y 2003, que solo tienen representatividad nacional y regional.

La pregunta relevante es qué indicadores de segregación utilizar y respecto a qué subgrupos de la población. Existe una gran cantidad de índices para medir segregación en la literatura, cada uno con sus ventajas y desventajas, pero optamos por dos en particular.

En primer lugar, con el objetivo de facilitar la comparación con otros trabajos, usamos el índice de disimilitud (Duncan \& Duncan, 1955), el más conocido y utilizado en la literatura de segregación. ${ }^{10}$ Este índice requiere que el investigador defina dos grupos y mida el porcentaje de un grupo que debe cambiarse de ubicación en cada subunidad geográfica, para que en esa subunidad el porcentaje de ese grupo sea el mismo que el que se da en la unidad geográfica superior (por ejemplo, el porcentaje de hogares de altos ingresos que tendrían que irse a vivir a otro barrio para obtener una distribución homogénea de hogares de altos ingresos en una ciudad). Toma valores entre 0 y 1 , donde 0 es integración total y 1 es segregación total.

$\mathrm{Al}$ igual que otros índices tradicionales de segregación, el índice de disimilitud es "no espacial", pues no considera la localización espacial de los grupos en la unidad geográfica. ${ }^{11}$ Adicionalmente, es relevante considerar que la segregación por ingresos es más difícil de identificar utilizando el índice de disimilitud, dado que el ingreso es una variable continua. Una solución a este problema, planteada por Watson (2009), es utilizar el Centile Gap Index (CGI), una medida que estima cuán distante está el ingreso de un hogar promedio en un área (una manzana o un distrito censal, por ejemplo), en términos de percentil, respecto al ingreso del hogar que está en la mediana de la distribución en la misma área metropolitana (la ciudad), comparado con qué tan distante estaría el mismo hogar si hubiera integración total. La fórmula matemática para el Centile Gap Index es la siguiente:

$$
C G I_{m}=\frac{\left(0.25-\left(\frac{1}{J_{m}}\right) \Sigma_{j}\left|P_{j}-P_{\text {medt }}\right|\right)}{0.25}
$$

donde $J m$ es el número de hogares en el área metropolitana m, $P j$ es el percentil de ingreso de hogares $\mathrm{j}$ en la distribución del ingreso del área metropolitana $\mathrm{m}, \mathrm{y}$ Pmedtj es el percentil de ingreso del hogar que está en la mediana de la distribución del ingreso en el área metropolitana $\mathrm{m}$. Si hubiera integración perfecta en un área metropolitana, cada área geográfica tendría la distribución completa de ingresos y el índice CGI sería igual a 0 . En el caso de segregación completa, el área metropolitana tendría solo barrios homogéneos y el índice CGI sería igual a $1 .^{12}$

10 Massey y Denton (1988) analizan y evalúan veinte índices de segregación distintos. Con base en el análisis de 60 áreas metropolitanas de Estados Unidos, concluyen que los índices de disimilitud y aislamiento superan a los demás en cuanto a la información que entregan.

11 Otra limitación del índice es el problema de checkerboard, que consiste en que las unidades geográficas pueden barajarse para obtener otra configuración espacial y el índice no cambia. Asimismo, el índice es sensible al tamaño de la unidad geográfica.

12 Una alternativa es utilizar el Rank-Order Information Theory Index (Reardon, 2009; Reardon \& Bischoff, 2011; Reardon \& O'Sullivan, 2004) que, al igual que el CGI, es insensible a cambios en la distribución del ingreso que mantienen el orden en el ranking de hogares. Sin embargo, 


\section{Resultados principales}

En esta sección se presentan los principales resultados obtenidos en la medición de segregación residencial por ingresos en el Gran Santiago para los años 1992 y 2002. A estos efectos se utiliza el ingreso de cada hogar, el cual es estimado usando las nuevas técnicas para combinar encuestas de hogares con datos del censo detalladas previamente, y se proyectan dos tipos de medidas de segregación.

En primer lugar, se presenta un conjunto de medidas de segregación basadas en el índice de disimilitud.

Trabajos previos que usan este índice para medir segregación residencial por estrato socioeconómico, lo han hecho a partir de identificar a los pobres o un grupo vulnerable versus el resto de la población. La segregación residencial de los pobres es sin duda interesante no solo descriptivamente, sino también porque la pobreza es el foco de políticas urbanas y sociales que suelen tener una alta prioridad para los gobiernos. Sin embargo, su medición por estrato es compleja, e incluso inconsistente, si se considera su evolución en el tiempo. En la década de los noventa, Chile experimentó una caída muy significativa de la pobreza absoluta. En la Región Metropolitana, entre 1992 y 2003, la pobreza cayó de 26,2\% a 13,1\%, es decir, se redujo a la mitad. En consecuencia, esta definición no corresponde a categorías que sean estables en el tiempo. Una alternativa es considerar un porcentaje fijo de la población de menores ingresos, la cual sin duda está entre los grupos más vulnerables de la población. En particular, se puede plantear que el 25\% más pobre en Chile durante todo este período -cercano a la tasa promedio nacional de pobreza en la década en cuestión- es un grupo que sin duda podemos asociar a un grado importante de carencias y falta de acceso a oportunidades.

Más allá de definir un umbral de vulnerabilidad adecuado, hay otras alternativas que deben ser consideradas. Por ejemplo, dada la importancia política y económica de las elites en la historia política y económica chilena, puede ser importante cuantificar la magnitud y la evolución de la segregación de los grupos más ricos. La importancia de la desigualdad en la parte alta de la distribución y sus implicancias para la cohesión social y la democracia se han puesto de relieve en la última década, lo cual hace que sea relevante analizarla (Atkinson, Picketty \& Saez, 2011; Bartels, 2009; Hacker \& Pierson, 2011; Picketty, 2014). ¿Hasta qué punto se encuentran espacialmente segregadas estas elites en Santiago, el centro económico y de poder más importante del país? En ese sentido, una partición natural de considerar en el análisis puede ser el $10 \%$ más rico. ${ }^{13}$

no captura la relación espacial de los hogares en el territorio, y su mayor complejidad no solo lo hace menos intuitivo, sino que además dificulta enormemente la estimación del error estándar del índice. Como el objetivo principal del trabajo es comparar resultados previos de la literatura con una estimación más precisa de los hogares que usa técnicas de última generación, optamos por estimar los mismos índices utilizados previamente e incorporar solo un índice nuevo con características ordinales.

13 En la Región Metropolitana, cerca de un 10\% de la matrícula escolar está en colegios particulares pagados y cerca de un $14 \%$ de la población -fuertemente concentrada en el decil de más altos ingresos- tiene cobertura de salud privada. El resto de la población asiste a escuelas subsidiadas y tiene cobertura de salud pública. 
Por último, hay otros grupos que son relevantes para las políticas públicas, especialmente en el ámbito de las finanzas públicas. Rodríguez (2001) plantea que la segregación en el Gran Santiago se debe, entre otros factores, a "los aspectos regresivos (a escala local) de la descentralización, en particular la relación directa entre recursos disponibles por los gobiernos locales y el nivel socioeconómico de sus residentes, que conduce a una inversión municipal per cápita mucho mayor en las comunas en donde residen los grupos de mayor ingreso" (p. 9), lo que hace relevante el estudio de la segregación de aquellos hogares que pagan contribuciones. En Chile, los municipios están a cargo de la oferta escolar municipal, los consultorios, ciertos aspectos de la seguridad ciudadana, la provisión de bienes públicos locales como las áreas verdes, la recolección de basura y el alumbrado de calles, junto a otras inversiones que se materializan en el espacio. Buena parte de las diferencias en los fondos disponibles para los municipios $-\mathrm{y}$, en consecuencia, para la provisión de bienes públicos- se debe a diferencias en la recaudación de contribuciones. A mediados de los años noventa, cerca de un $36 \%$ de la población -el sector de más altos ingresos, típicamente- pagaba contribuciones.

Por las razones anteriores, se analizan y presentan medidas de segregación para los tres "grupos minoritarios" mencionados respecto al resto de la población:

- pobres (partición 25 más pobre, 75 restante),

- altos ingresos (partición 10 más rico, 90 restante),

- contribuyentes (partición 64 más pobre, 36 restante).

Con el objeto de hacer más extensivo el análisis y menos arbitrario el punto de corte en la distribución de ingresos, se presentan también estimaciones de segregación para grupos cuyos cortes están definidos por cada decil. Vale decir, dos grupos a partir del $10 \%$ más pobre y el $90 \%$ más rico, el $20 \%$ más pobre y el $80 \%$ más rico, y así sucesivamente hasta el $90 \%$ más pobre y el 10\% más rico. Esto permite, por una parte, comparar la segregación asociada a grupos más vulnerables respecto a la de los más ricos y, al mismo tiempo, establecer comparaciones robustas en el tiempo.

Adicionalmente, se presenta un conjunto de medidas de segregación basadas en el Centile Gap Index (CGI) que, a diferencia del índice de disimilitud, está diseńado para una distribución continua, como el ingreso.

En el cuadro 1 se presentan las estimaciones de los índices de segregación para 1992 y 2002. En el caso del índice de disimilitud, se reportan los índices para los tres grupos mencionados: $25 \%$ más pobre, contribuyentes y $10 \%$ más rico, con sus respectivos errores estándar.

A partir de los resultados, hay cuatro hechos estilizados centrales:

Hecho estilizado 1: se observa que la segregación basada en cortes de vulnerabilidad y contribuyentes aumentó entre 1992 y 2002. Para la medida de segregación de los más ricos, no hay diferencias estadísticamente significativas entre 1992 y 2002. Por otra parte, el índice de segregación CGI también registró un aumento estadísticamente significativo en este período. 
Hecho estilizado 2: la segregación de los más ricos es más alta que la de los grupos más vulnerables tanto para 1992 como para 2002. El índice de disimilitud basado en el 10\% más rico es estable en el tiempo, alcanzando un valor de 0,53; es decir, para lograr niveles de integración ideales, más de la mitad de los hogares pertenecientes a este grupo debería migrar a otros barrios.

CUADRo I | Segregación en el Gran Santiago con distintos índices: CGI y Disimilitud (1992 y 2002)

\begin{tabular}{|l|c|c|c|}
\hline \multicolumn{1}{|c|}{ ÍNDICES } & I992 & $\mathbf{2 0 0 2}$ & $\begin{array}{c}\text { DIFERENCIA } \\
\text { I992 / 2002 }\end{array}$ \\
\hline \multirow{2}{*}{ Índice CGI } & 0,1917 & 0,1991 & $0,0074^{* *}$ \\
\cline { 2 - 4 } & $(0,0171)^{*}$ & $(0,0103)^{*}$ & \\
\hline Índice de disimilitud para distintas particiones & 0,3381 & 0,3619 & $0,0238^{* *}$ \\
\hline \multirow{2}{*}{ Disimilitud pobreza (25-75)*** } & $(0,0146)^{*}$ & $(0,0084)^{*}$ & \multicolumn{2}{|c|}{} \\
\cline { 2 - 4 } & 0,3774 & 0,3998 & $0,0224^{* *}$ \\
\hline \multirow{2}{*}{ Disimilitud contribuyentes (64-36) } & $(0,0188)^{*}$ & $(0,0123)^{*}$ & $-0,0023$ \\
\cline { 2 - 4 } & 0,5317 & 0,5294 & $(0,0173)^{*}$ \\
\hline \multirow{2}{*}{ Disimilitud altos ingresos (90-10) } & $(0,0285)^{*}$ & & \\
\cline { 2 - 4 } &
\end{tabular}

NOTAS

$\left(^{*}\right)$ DESVIACIÓN ESTÁNDAR, ENTRE PARÉNTESIS, CORRESPONDEN A IOO ITERACIONES DE LA **) DISTRIBUCIÓN DE INGRESOS POR HOGAR.

$\left.{ }^{* * *}\right)$ DISIMILITUd POBREZA CONSIDERA LA SEGREGACIÓN ENTRE EL QUINTIL MÁS POBRE Y EL RESTO DE LA POBlaCión; CONTRIBUYENTES, ENTRE EL 64\% MÁS POBRE Y EL 36\% MÁs RiCO; ALTOS INGRESOS, ENTRE EL IO\% MÁS RICO Y EL RESTO DE LA POBLACIÓN.

FUENTE ELABORACIÓN PROPIA

El cuadro 2 recoge una comparación de los niveles de segregación encontrados para Santiago, respecto de mediciones para ciudades norteamericanas. Independientemente de la medida utilizada, la segregación en Santiago es muy superior al promedio de los registros para ciudades norteamericanas usando CGI, y niveles algo mayores en el caso de la disimilitud a partir del grupo de pobreza.

CUADRO 2 Comparación Chile y EE.UU. para distintos índices

\begin{tabular}{|l|c|l|}
\hline ÍNDICE DE SEGREGACIÓN & $\begin{array}{c}\text { REGIÓN } \\
\text { METROPOLITANA } \\
\mathbf{2 0 0 2}\end{array}$ & \multicolumn{1}{c|}{ COMPARACIÓN CON EE.UU. } \\
\hline Centil Gap Index 2002 & 0,199 & $\begin{array}{l}\text { Promedio 221 áreas metropolitanas: } \\
1970=0,110 ; 2000=0,120 \text { (Watson, 2009). }\end{array}$ \\
\hline $\begin{array}{l}\text { Disimilitud pobreza } \\
(25 / 75)\end{array}$ & 0,362 & $\begin{array}{l}\text { Promedio 331 áreas metropolitanas: 0,351 (Iceland } \\
\text { \& Wilkes, 2006). }\end{array}$ \\
\hline $\begin{array}{l}\text { Disimilitud altos ingresos } \\
(90 / 10)\end{array}$ & 0,532 & $\begin{array}{l}\text { Promedio segregación racial para 298 áreas metropo- } \\
\text { litanas: 0,529 (Wilkes \& Iceland, 2004). }\end{array}$ \\
\hline
\end{tabular}

FUENTE ELABORACIÓN PROPIA 
Si bien no existen estudios directamente comparables para América Latina, algunos han mostrado que los niveles de segregación en Santiago son comparables a los de otras metrópolis latinoamericanas (Lambiri \& Vargas, 2011; Rodríguez \& Arriagada, 2004), si bien más altos que en México tanto para ingresos altos como bajos (Monkkonen, 2012) y menores para áreas metropolitanas en Brasil (Telles, 1995).

Hecho estilizado 3: independientemente de la medida utilizada y el año, los niveles de segregación residencial de ingresos del Gran Santiago son considerablemente más altos que la segregación residencial de ingresos de las áreas metropolitanas en Estados Unidos.

Hecho estilizado 4: la segregación residencial de ingresos del Gran Santiago asociada el decil más rico de la distribución de ingresos, de 0,532, exhibe niveles de segregación comparables con los niveles de segregación residencial racial detectados en estudios de áreas metropolitanas de Estados Unidos. Si bien el nivel de segregación no se compara con los mayores niveles de segregación racial en Estados Unidos (0,84 en Detroit o 0,77 en Chicago), se aproxima bastante al promedio.

FIGURA I | Índice de disimilitud para distintos grupos, cortes por decil (1992 y 2002)

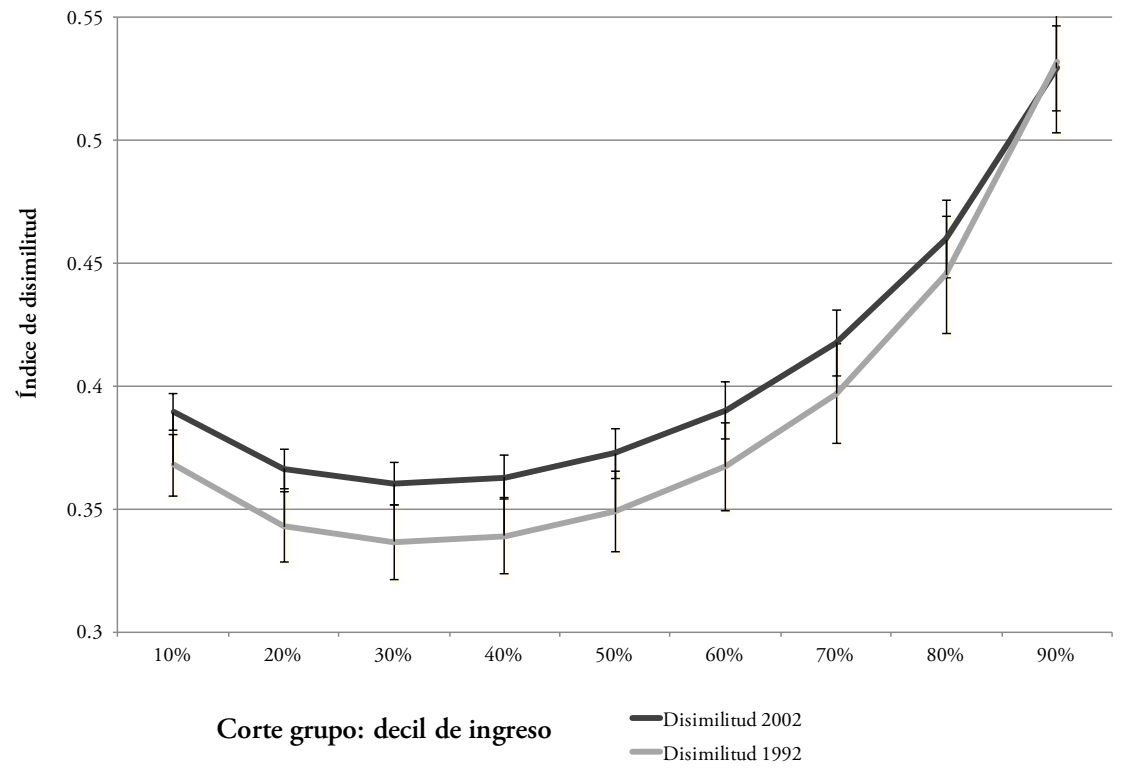

FUENTE ELABORACIÓN PROPIA

En suma, a diferencia de lo señalado por otros estudios, los resultados muestran que la segregación residencial por ingresos en Santiago aumentó entre 1992 y 2002, exceptuando la segregación asociada al decil más rico, que se ha mantenido estable 
en un alto nivel. La segregación del decil más alto es sistemáticamente mayor que la de grupos vulnerables. Las medidas de segregación para Santiago son mayores que las obtenidas para ciudades norteamericanas.

\section{Contribución comunal a la segregación del Gran Santiago}

Si bien son relevantes e informativos los resultados presentados de segregación para el área metropolitana de Santiago y su comparación con otras mediciones de segregación en otras ciudades en el mundo, es importante realizar un análisis de la segregación a escalas territoriales menores. ${ }^{14}$ Por ello, a continuación se presentan los resultados asociados a la descomposición de la segregación a nivel comunal con una focalización en el índice CGI.

El ejercicio entrega una desagregación espacial de la segregación de la ciudad, teniendo como foco explorar la heterogeneidad comunal de la contribución a la segregación. Sin duda existe cierta arbitrariedad al escoger la comuna como escala de desagregación, en lugar de manzanas o unidades vecinales, que podrían dar cuenta de patrones de segregación que atraviesan la frontera entre comunas. Sin embargo, estas últimas son unidades administrativas relevantes en sí mismas, ya que las contribuciones, la provisión de bienes y servicios públicos locales, así como la implementación de muchas políticas públicas y el diseño de los planes reguladores de desarrollo inmobiliario, caen en la jurisdicción municipal. Finalmente, en períodos de tiempo considerable, las ciudades experimentan importantes cambios en su morfología, se expanden y contraen sus límites. Frente a estos cambios, las comunas son "geográficamente estables" al paso del tiempo.

\section{Descomposición comunal del CGI}

El índice CGI puede descomponerse como un promedio ponderado de la contribución de cada comuna $j$ al índice, donde los ponderadores son simplemente la fracción de la población comunal sobre la población total del Gran Santiago:

$$
C G I_{\text {Santiago }}=\sum_{j: c o m u n a} \alpha_{j} C G I_{\text {Santiago }, j}
$$

donde $\alpha_{j}=$ \#Hogares de Comunaj/\#Hogares de Santiago mide el tamaño relativo de la comuna $j$ con respecto a la ciudad y, en línea con la definición del CGI en la sección previa,

$$
C G I_{\text {Santiago }, j}=\left(0.25-1 / J_{S} \sum_{\text {h:hogaren } j}\left|P_{h}-P_{\text {med, }, h}\right|\right) / 0.25
$$

14 Estos resultados no se hacen cargo del debate en la literatura de estudios urbanos sobre cuál es la escala relevante para describir y medir la segregación; ello requiere un tratamiento metodológico específico y un foco distinto, que escapa a la contribución de este artículo. 
es la contribución específica de los hogares en la comuna $j$ al índice CGi de la ciudad. Esta contribución de la comuna $j$ a la segregación no mide la segregación dentro de la comuna, ya que, para cada manzana, el índice considera desviaciones de la distribución del ingreso de cada una respecto a la distribución del ingreso de la ciudad y no la comuna.

Una interpretación de esta contribución comunal es que es una medida que captura el promedio comunal de la diferencia entre la distribución de ingresos de una manzana y la distribución de la ciudad. Esta cantidad permite, en conjunto con los cambios poblacionales, evaluar cuál sería el impacto "incremental” sobre la medida de segregación del Gran Santiago de ignorarse una comuna.

Los cambios en el tiempo de la contribución comunal entregan, a su vez, información relevante sobre la dinámica social y espacial de las comunas. Las variaciones en el tiempo de esta contribución permiten comprender qué comunas se han vuelto más o menos integradas: ello está dado no solo por esta contribución comunal, sino también por cambios en los pesos de cada comuna asociados con cambios en la población comunal relativa al resto de la ciudad. ${ }^{15}$

\section{FIGURA 2 | Contribución comunal CGI 2002 y cambios 1992-2002}

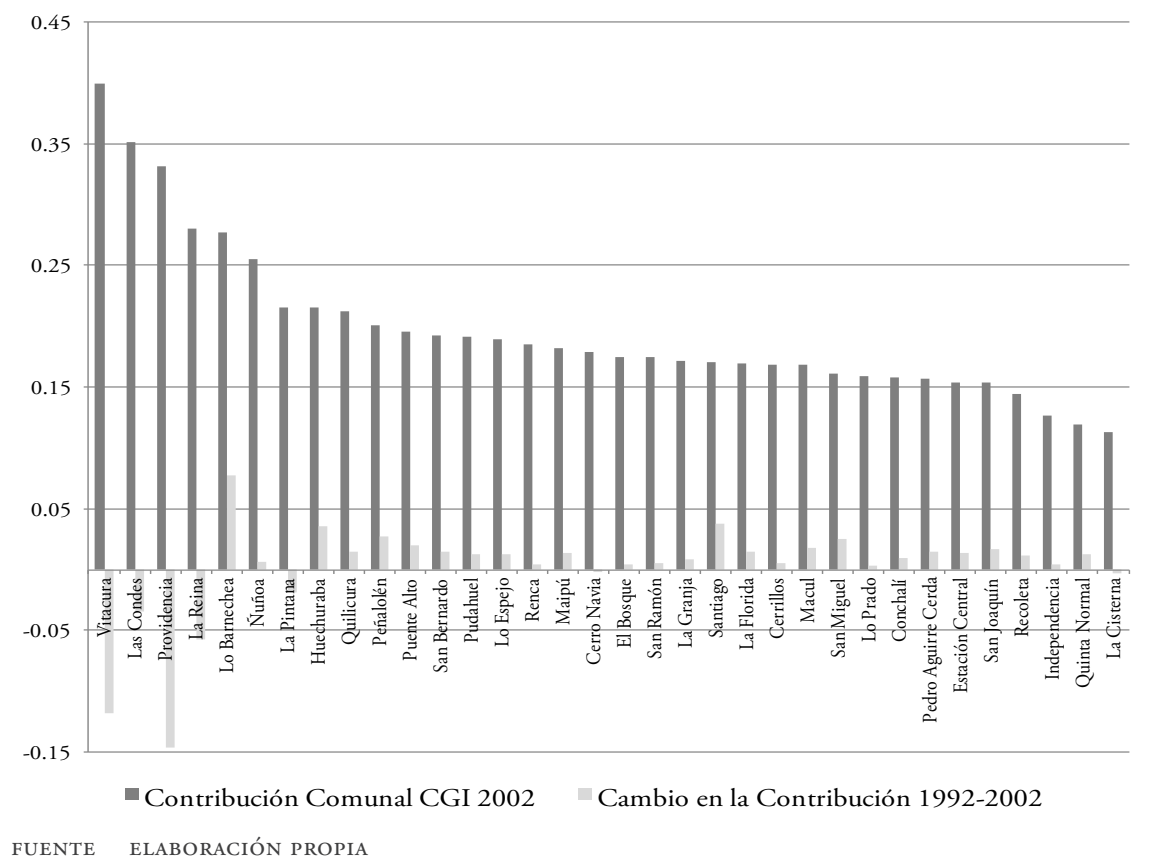

15 El cuadro A1 en el apéndice presenta la contribución comunal de cada una de las 34 comunas del Gran Santiago para los años 1992 y 2002, los cambios entre estas dos mediciones y los errores estándar de cada medida. 
La figura 2 muestra tanto los niveles de la contribución comunal como los cambios entre 1992 y 2002. Se observa una gran heterogeneidad en ambas variables. Las comunas que constituyen el "barrio alto" de la ciudad -Vitacura, Las Condes, Providencia, La Reina, Nunnoa, y más recientemente Lo Barnechea- son precisamente las que exhiben una mayor contribución específica a la segregación de la ciudad, con una contribución promedio de 0,316 que contrasta con el promedio de 0,172 para el resto de las comunas del Gran Santiago. A su vez, Vitacura, Las Condes, Providencia y La Reina, son las comunas que más reducen su contribución a la segregación de la ciudad en la década analizada, mientras que Lo Barnechea la aumenta. Es decir, parece existir una correlación negativa entre el nivel de la contribución y el cambio en ella.

CUADRO 3 | Comunas que redujeron su contribución al CGI $\left(\right.$ CGI $\left._{\text {Santiago,j }}<0\right)$

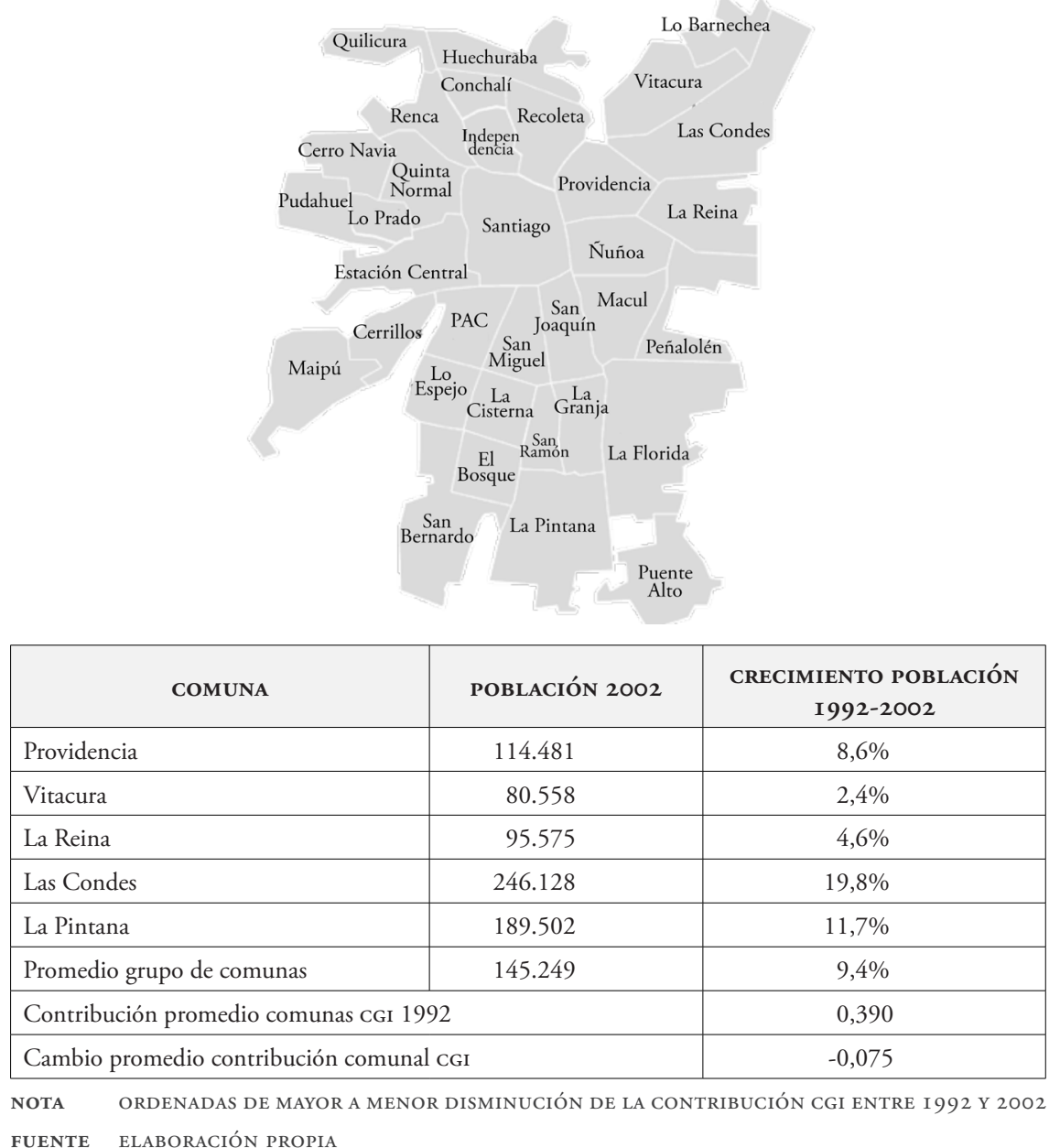


De las 34 comunas del Gran Santiago, 20 aumentaron su contribución de segregación significativamente y solo 5 comunas lo disminuyeron también significativamente. Las comunas restantes tuvieron cambios moderados y estadísticamente no importantes (7 aumentan y 2 disminuyen su contribución). En los cuadros 3 y 4 se reportan los niveles de segregación en 1992 y los cambios promedio, así como el crecimiento poblacional promedio para dos grupos de comunas. El primero corresponde a las cinco comunas que experimentaron una caída en la contribución a la segregación entre 1992 y 2002, ordenadas de mayor a menor disminución. Lideran el grupo cuatro del "barrio alto" de Santiago -Providencia, La Reina, Vitacura y Las Condes- que, en línea con lo señalado por Sabatini et al. (2010), disminuyeron su contribución a la segregación. ${ }^{16}$ Sin embargo, se trata de las comunas que más contribuyen al índice de segregación. En promedio, la caída de la contribución de estas comunas al CGI fue -0,075. Con la excepción de Las Condes, cuya población aumentó casi un $20 \%$ entre ambos censos, las restantes cuatro comunas experimentaron tasas de crecimiento poblacional menores que la tasa de crecimiento del Gran Santiago durante el período, que ascendió a un 13,6\%.

CUADRO 4 | Comunas que aumentaron su contribución al CGI $\left(\right.$ CGI (Santiago,j) $\left._{\text {. }}>0.07\right)$

\begin{tabular}{|l|c|c|c|}
\hline \multicolumn{1}{|c|}{ COMUNA } & POBLACIÓN 2002 & $\begin{array}{c}\text { CRECIMIENTO } \\
\text { POBLACIÓN } \\
\text { I992-2002 }\end{array}$ & $\begin{array}{c}\text { AUMENTO EN } \\
\text { CONTRIBUCIÓN } \\
\text { AL CGI }\end{array}$ \\
\hline Lo Barnechea & 73.127 & $55,3 \%$ & 0,0772 \\
\hline Santiago & 184.709 & $-14,2 \%$ & 0,0382 \\
\hline Huechuraba & 73.965 & $19,8 \%$ & 0,0353 \\
\hline Peńalolén & 215.593 & $20,4 \%$ & 0,0274 \\
\hline San Miguel & 75.222 & $-5,1 \%$ & 0,0247 \\
\hline Puente Alto & 488.796 & $95,0 \%$ & 0,0201 \\
\hline San Joaquín & 96.203 & $-15,1 \%$ & 0,0168 \\
\hline Macul & 11.000 & $-7,2 \%$ & 0,0175 \\
\hline San Bernardo & 244.564 & $30,1 \%$ & 0,0145 \\
\hline Quilicura & 126.396 & $207,6 \%$ & 0,0144 \\
\hline Pedro Aguirre Cerda & 114.211 & $-12,3 \%$ & 0,0144 \\
\hline Maipú & 466.440 & $82,1 \%$ & 0,0136 \\
\hline La Florida & 364.070 & $10,7 \%$ & 0,0144 \\
\hline Estación Central & 123.939 & $-10,0 \%$ & 0,0135 \\
\hline Quinta Normal & 103.051 & $-10,9 \%$ & 0,0123 \\
\hline Pudahuel & 195.005 & $42,1 \%$ & 0123 \\
\hline
\end{tabular}

(continúa)

16 En contraste con estos autores, este trabajo muestra que este efecto no fue dominante y que parte de la población de más altos ingresos se desplazó a comunas suburbanas como Lo Barnechea. 
(continuación)

\begin{tabular}{|l|c|c|c|}
\hline \multicolumn{1}{|c|}{ COMUNA } & POBLACIÓN 2002 & $\begin{array}{c}\text { CRECIMIENTO } \\
\text { POBLACIÓN } \\
\text { I992-2002 }\end{array}$ & $\begin{array}{c}\text { AUMENTO EN } \\
\text { CONTRIBUCIÓN } \\
\text { AL CGI }\end{array}$ \\
\hline Lo Espejo & 112.500 & $-6,3 \%$ & 0,0123 \\
\hline Recoleta & 145.694 & $-10,5 \%$ & 0,0115 \\
\hline Conchalí & 132.823 & $-13,0 \%$ & 0,0100 \\
\hline La Granja & 132.154 & $-0,5 \%$ & 0,0081 \\
\hline Promedio grupo de comunas & 178.973 & $22,9 \%$ & 0,159 \\
\hline Contribución promedio comunas CGI 1992 & & 0,020 \\
\hline Cambio promedio contribución comunal CGI & & \\
\hline
\end{tabular}

NOTA ORDENADAS DE MAYOR A MENOR AUMENTO SIGNIFICATIVO DE LA CONTRIBUCIÓN CGI ENTRE I 992 Y 2002

FUENTE ELABORACIÓN PROPIA

El segundo grupo consiste en las 20 comunas cuya contribución al CGI creció más que el aumento total del CGI entre 1992 y 2002 en el Gran Santiago (0,074, véase cuadro 1). ${ }^{17}$ Las comunas se ordenan de mayor a menor aumento en la contribución. Lidera el grupo Lo Barnechea, que en este período se consolidó como un suburbio de altos ingresos. En promedio, el aumento de la contribución CGI en este grupo fue de 0,020. En él se incluyen las comunas más pobladas de Santiago y del país -Puente Alto y Maipú-y, en promedio, el grupo experimentó un aumento poblacional de un 22,9\%, valor bastante mayor que el promedio del Gran Santiago, de un 13,6\%.

Sin embargo, hay bastante heterogeneidad en el grupo de comunas. Por una parte, diversos municipios relativamente centrales y cercanos entre sí, como Santiago, San Joaquín, Lo Espejo, Pedro Aguirre Cerda, San Miguel y Macul, redujeron su población entre un 5\% y 15\%, aproximadamente. Todas ellas se encuentran localizadas al sur de la comuna de Santiago, limítrofes entre sí y en relación con la avenida circunvalación Américo Vespucio. Otras comunas que tuvieron pérdidas de población son Conchalí, Quinta Normal, Recoleta y Estación Central, que redujeron su población entre $13 \%$ y $10 \%$. Todas ellas están localizadas al norte y poniente de la comuna de Santiago. Por otra parte, otras más periféricas, como Quilicura, Puente Alto, Maipú, Pudahuel y San Bernardo, experimentaron aumentos poblacionales extraordinarios, de $208 \%, 95 \%, 80 \%, 42 \%$ y $30 \%$, respectivamente, sumando un total cercano a los 620.000 habitantes. Comunas más pequeñas, como Lo Barnechea y Huechuraba también crecieron considerablemente, un 55\% y 20\%, respectivamente. Otra comuna cuya población creció por sobre el promedio metropolitano, en torno al $20 \%$, fue Peñalolén.

\section{Observaciones espaciales de la segregación}

Los cambios demográficos comunales sugieren un trasfondo espacial complementario a las mediciones efectuadas. Más allá del efecto mecánico de los cambios poblacionales sobre el índice CGI (a través de los ponderadores), la masividad y

17 Todos estos cambios son estadísticamente significativos. 
naturaleza de los flujos residenciales está fuertemente asociada con los cambios en la segregación. Es más, dichas variaciones no ocurren en lugares arbitrarios de la ciudad.

La figura 3 muestra dos cartografías con la descomposición comunal del CGI para los años 1992 y 2002 en la ciudad de Santiago. Es posible distinguir un aumento de la contribución a la segregación en barrios céntricos como Santiago, San Miguel y Quinta Normal. Así también, en torno a barrios ya con alta segregación como Las Condes y Vitacura en 1992, otros barrios vecinos como Lo Barnechea y Peñalolén -respectivamente en el sector nororiente y Quilicura en el norponiente- suman significativas contribuciones a la segregación. Hay, además, una expansión de los límites de la ciudad, tanto en el límite sur de Puente Alto como en los límites norte de Huechuraba y Quilicura, para mencionar solo las principales áreas de expansión urbana.

\section{FIGURA 3 | Mapa desagregación comunal índice CGI (1992 y 2002)}
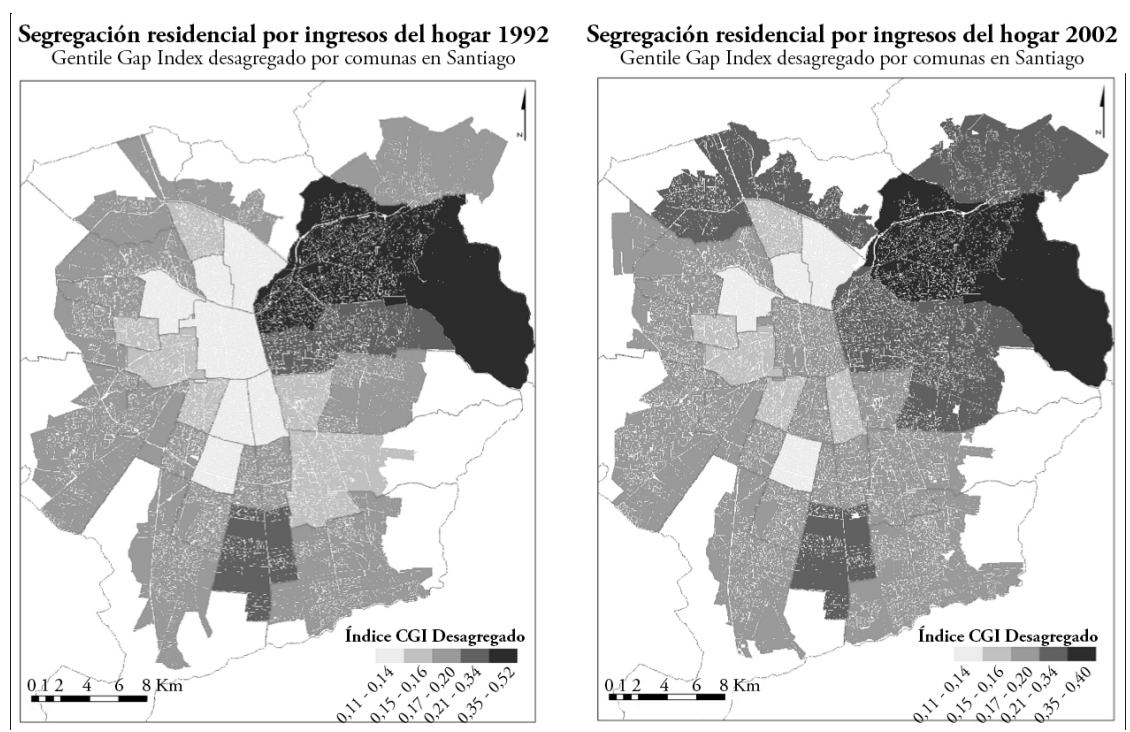

FUENTE ELABORACIÓN PROPIA

En general, destaca la confirmación de tendencias urbanas de segregación ya expuestas en estudios anteriores (Sabatini et al., 2001; Poduje, 2011). La primera: la contribución de segregación de altos ingresos hacia el sector nororiente de la ciudad, tal como se muestra en el mapa de 1992 de la figura 4. Sin embargo, el mapa de 2002 hace evidente la cantidad de comunas que incrementan su CGI, como Peñalolén, Lo Barnechea, Huechuraba y Quilicura, frente a las anteriores comunas con los más altos CGI, como Vitacura, Las Condes, La Reina, Providencia y Nuńoa. Notoriamente, todas estas sobrepasan el promedio CGI de la ciudad, el cual prácticamente mantiene su contribución de segregación entre 1992 y 2002. La segunda tendencia confirmada 
es que la contribución a la segregación también aumenta en comunas pericentrales. ${ }^{18}$ De las diez comunas colindantes con el municipio de Santiago, un 60\% aumenta su contribución de segregación significativamente, un 30\% lo aumenta moderadamente y solo una comuna lo baja (Providencia).

Estos resultados sugieren una potencial asociación entre la contribución comunal a la segregación y los planes de regulación urbana implementados por cada comuna, los cuales están fuertemente basados en la zonificación de suelo urbano, al establecer los tipos de usos de suelo y las condiciones de edificación en ellos. Dos condiciones que pueden impactar el CGI son la definición de superficie predial mínima y de la máxima densidad habitacional. ${ }^{19} \mathrm{Y}$ ambas inciden fuertemente en las características de la oferta inmobiliaria de una comuna y en el acceso a propiedades en ella según nivel socioeconómico.

FIGURA 4 Mapas de pobreza y riqueza con \% quintil más pobre por manzana (1992 y 2002)

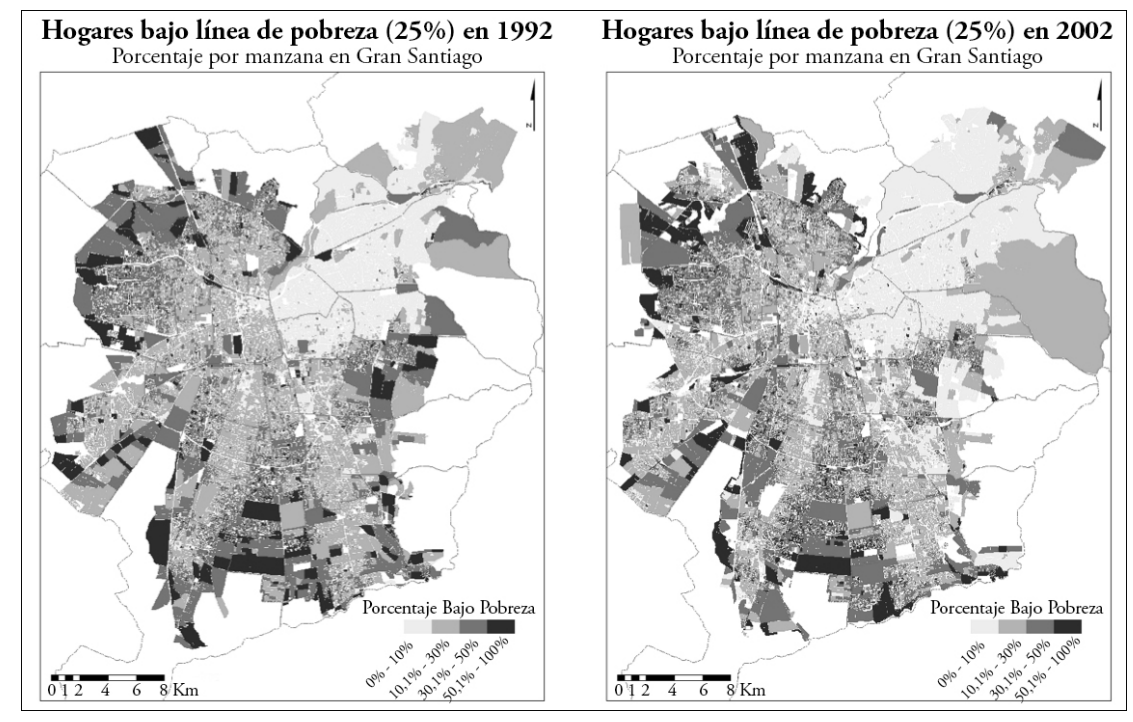

FUENTE ELABORACIÓN PROPIA

Es interesante contrastar esta cartografía con la de la figura 4. Esta última muestra mapas de pobreza -entendida como la localización del quintil más pobre en el espacio- y del decil más rico en unidades espaciales diferentes a las comunas. En lugar de utilizar comunas como en la figura 3, se utiliza una unidad espacial funcional a la ciudad: la manzana urbana o el conjunto de lotes de propiedad rodeados por vialidad. Esta representación refina el análisis espacial de la segregación, pues permite: i) representar la segregación en una escala espacial menor a

18 Denominación común a municipios cercanos y aledańos a centros históricos urbanos.

19 Una establece el tamańo menor al cual se puede subdividir un terreno y, por ende, fija un valor de mercado para este; y la otra establece la población por unidad de área permitida en cada sector de la comuna. 
la comuna; ii) vincular la segregación a otros componentes urbanos importantes, como, por ejemplo, transporte y geografía; y iii) establecer una correspondencia entre la contribución local de la segregación con densidad poblacional en esas áreas.

La figura 4 ilustra aumentos de pobreza en los barrios céntricos y, a su vez, una disminución de la presencia del decil más rico en comunas aledañas, como Providencia. Esto hace que el eje central de la ciudad -compuesto por el Centro histórico y civil hasta el Centro de negocios en Providencia y Las Condes- se mantenga con una alta segregación de altos ingresos, siguiendo las tendencias de desarrollo urbano para ciudades en la región (Borsdorf, 2003); o, en el caso de la cartografía mencionada, con un porcentaje menor de población de bajos ingresos.

Respecto a las tendencias de localización de altos ingresos, entre 1992 y 2002 la pobreza retrocede y aumenta la presencia del decil más rico en Lo Barnechea y sectores precordilleranos de Las Condes, La Reina y Peñalolén. Es decir, el oriente precordillerano tiende a homogeneizarse y la expansión en esa zona de la ciudad se asocia con una localización de hogares de altos ingresos. ${ }^{20}$ Por su parte, las expansiones de la ciudad en la periferia del nororiente y sur de Santiago son extensiones de pobreza.

El aumento poblacional de Puente Alto -238.000 habitantes adicionales entre 1992 y 2002- se debe en buena medida a la construcción masiva de condominios de vivienda social en la década de los noventa. Los condominios sociales de Bajos de Mena, uno de los guetos urbanos más extensos de Santiago (Poduje, 2011), se entregaron entre 1995 y 1997 y concentran cerca de 122.000 habitantes, es decir, explica más de la mitad del crecimiento de Puente Alto en ese periodo. Algo similar ocurre en Quilicura, cuya población creció en cerca de 85.000 habitantes entre 1992 y 2002. Sectores como la población Parinacota o Lo Marcoleta-El Mañío, también reconocidos como guetos urbanos, concentran más de 60.000 habitantes. Es decir, nuestra desagregación comunal es consistente con la hipótesis de una política de vivienda social masiva asociada a la sobredensificación de sectores periféricos con bajos precios de suelos, que se asocia a contribuciones importantes a la segregación a través de la concentración de hogares de ingresos bajos.

Los mapas en la figura 5 muestran el porcentaje de localización con manzanas del $10 \%$ de mayor ingreso. Se evidencian dos tendencias espaciales entre 1992 y 2002 : la ampliación del cono nororiente de localización de altos ingresos y la consolidación de corredores sur de localización de mayores ingresos.

Respecto a la primera tendencia, la desagregación comunal permite vislumbrar la potencial relación entre algunas dinámicas inmobiliarias asociadas a los barrios altos de la ciudad de Santiago y sus comunas circundantes. Durante esta década, sectores de Peñalolén, Huechuraba y Lo Barnechea -en los cuales buena parte de la oferta corresponde a condominios extensos y cerrados- atraen una demanda de sectores de ingresos altos, algo consistente con los aumentos en la contribución a la segregación que identifica nuestra desagregación espacial.

Por una parte, se muestra claramente que la localización de altos ingresos en el "cono" nororiente de la ciudad se expande durante la década de los noventa hacia

20 La única excepción de algunas manzanas que aumentaron la localización de bajos ingresos es por proyectos de mejora de vivienda social en Lo Barnechea. En parte, se debe a los efectos de conurbación a partir de la expansión urbana de Santiago con el histórico poblado de Lo Barnechea, antes desconectado de la ciudad. 
los sectores sur con características similares de la precordillera, y también a sectores norte de la ciudad, de características geográficas parecidas. Por otra parte, es una década de gran densificación de las comunas del barrio alto tradicional -Providencia, Las Condes, La Reina y Vitacura-, lo que podría explicar la disminución de la contribución a la segregación de estas comunas, según lo indican nuestros datos.

Aunque se requiere un análisis con microdatos que identifique los desarrollos inmobiliarios de la época, esta desagregación espacial es consistente con un patrón complejo, que podría incluir desplazamientos en el espacio de sectores consolidados a sectores nuevos, la precordillera o nuevos suburbios, que pueden contribuir a una mayor segregación. Y asimismo, a una densificación que podría reducirla. En conjunto, el tamaño de los cambios en estas comunas es bastante menor que el de los asociados a otras como Quilicura, Puente Alto o San Bernardo. ${ }^{21}$

FIGURA 5 | Mapas de pobreza y riqueza con \% del decil más rico por manzana (1992 y 2002)

Hogares en el decil más alto de ingreso en 1992 Hogares en el decil más alto de ingreso en 2002 Porcentaje por manzana en Gran Santiago

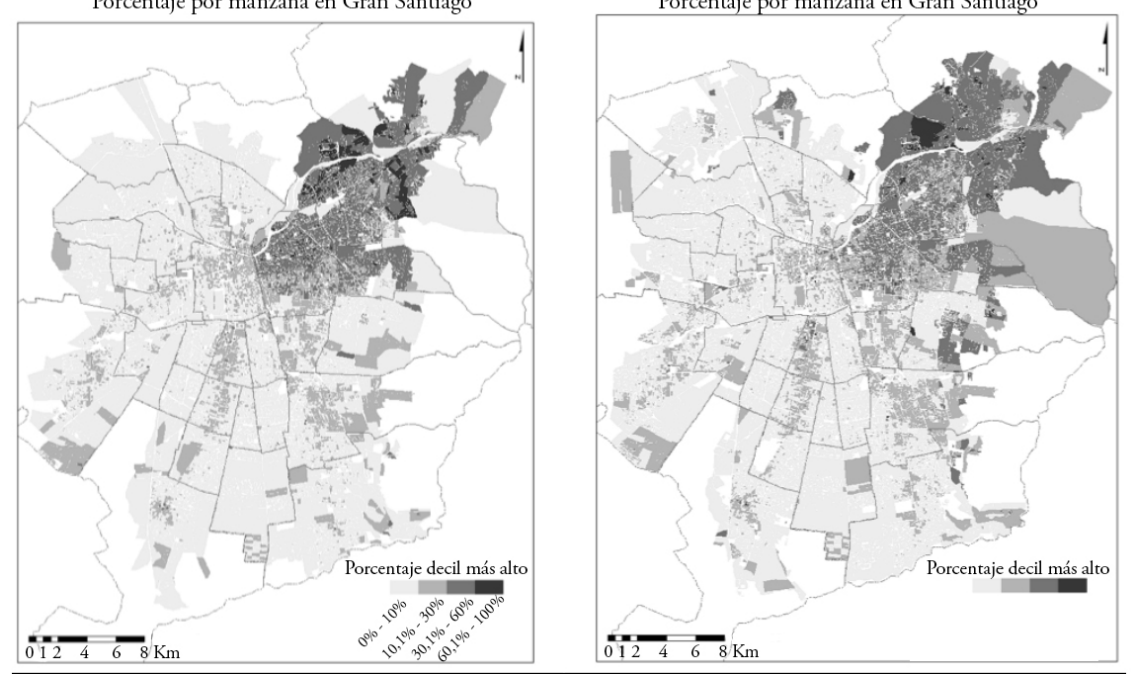

FUENTE ELABORACIÓN PROPIA

Respecto a la segunda tendencia espacial, la existencia de corredores de desarrollo urbano ya se evidencia en el mapa de 1992 en la figura 5: se trata de los corredores correspondientes a las avenidas Pajaritos, Gran Avenida, Vicuña Mackenna y La Florida, en orden de poniente a oriente. De estas, las tres primeras han tenido fuertes inversiones en infraestructuras de transporte público. A su vez, las dos tendencias recién explicadas muestran de mejor manera las contribuciones de segregación utilizando la unidad espacial de la manzana, dada la heterogeneidad dentro de cada comuna y las relaciones de desarrollo urbano entre ellas.

21 El crecimiento poblacional en Lo Barnechea y en Peńalolén, sumado en el periodo analizado, es de 63.000 habitantes aproximadamente, mientras que Providencia, La Reina, Vitacura y Las Condes suman de cerca de 56.000 . 


\section{Conclusión}

Este trabajo presenta mediciones de segregación residencial por ingresos para el Gran Santiago en 1992 y 2002. La metodología utilizada, basada en estimaciones de ingreso por hogar que combinan encuestas de panel de gran escala con información censal, se hace cargo de algunas de las limitaciones que tienen mediciones de segregación previas. Adicionalmente, se utiliza un índice de segregación más apropiado para capturar diferencias entre niveles de ingreso, mientras la estimación de los errores estándar de cada índice hace posible discriminar estadísticamente entre dos mediciones distintas.

Los resultados muestran que los hogares de ingresos altos están más aislados que los hogares pobres, con niveles comparables a la segregación residencial racial en Estados Unidos. Sin embargo, la segregación medida con el índice CGI -que permite capturar toda la heterogeneidad de la distribución del ingreso local- es mucho mayor. Los datos también muestran que la segregación en Santiago aumentó en la década transcurrida entre 1992 y 2002. Debido a que el método genera errores estándar para todos los índices de segregación, nuestros resultados sobre el cambio de la segregación en el tiempo permiten afirmar que las variaciones son estadísticamente significativas y no simplemente fluctuaciones en el margen de error. El hecho de que los resultados difieran parcialmente de estudios previos refuerza la importancia de contar con métodos robustos para estimación de la segregación residencial.

Más allá del aumento de dicha segregación, la ciudad manifiesta diferencias sustanciales entre comunas. Los cambios poblacionales y la expansión de la ciudad sugieren que la dinámica de la segregación residencial se asocia a dinámicas inmobiliarias y a la política de vivienda durante este período. Cuantificar los efectos del funcionamiento del mercado inmobiliario y las políticas públicas de gran escala en la ciudad sobre la segregación es una materia relevante para futuras investigaciones. Esto podría requerir modelos de segregación y datos en una escala micro para identificar mejor el impacto que tienen en ciertas comunas factores como la densificación y las políticas urbanas y de vivienda, entre otros, y el efecto de inversiones en infraestructura, como la expansión de las líneas de metro o la construcción de carreteras urbanas.

Utilizar la misma metodología para evaluar la evolución de la segregación en otras ciudades de Chile es también materia de investigación futura, así como estudiar la asociación entre segregación residencial y variables sociales, como patrones de criminalidad o la desigualdad social. Finalmente, hay aspectos metodológicos que van más allá de los objetivos de este trabajo y que por ello no se abordan. En particular, sería importante medir segregación utilizando el Rank-Order Information Theory Index (Reardon, 2009) y avanzar en el desarrollo y la implementación de índices de segregación que sean sensibles a la relación espacial entre unidades geográficas de menor escala. 


\section{Referencias bibliográficas}

Agostini, C. (2010). Pobreza, desigualdad y segregación en la región metropolitana. Estudios Públicos, (117), 219-268. En http://www.cepchile.cl/dms/archivo_4600_2749/ rev117_agostini.pdf

Agostini, C. \& Brown, P. (2007). Desigualdad geográfica en Chile. Revista de Análisis Económico, 22 (1), 3-33. En http://www.rae-ear.org/index.php/rae/article/view/65/125

Agostini, C. A. \& Brown, P. H. (2010a). Local distributional effects of government cash transfers in Chile. Review of Income and Wealth, 56 (2), 366-388. doi: 10.1111/j.14754991.2009.00373.x

Agostini, C. A. \& Brown, P. H. (2010b). Inequality at low levels of aggregation in Chile. Review of Development Economics, 14 (2), 213-226. doi: 10.1111/j.1467-9361.2010.00548.x

Agostini, C. A. \& Brown, P. H. (2011). Cash transfers and poverty reduction in Chile. Journal of Regional Science, 53 (3), 604-625. doi: 10.1111/j.1467-9787.2010.00707.x

Agostini, C. A., Brown, P. H. \& Góngora, D. P. (2008). Distribución espacial de la pobreza en Chile. Estudios de Economía, 35 (1), 79-110. En http://dx.doi.org/10.4067/S071852862008000100005

Atkinson, A., Picketty, T. \& Saez, E. (2011). Top incomes in the long run of history. Journal of Economic Literature, 49 (1), 3-71. En http://eml.berkeley.edu/ -saez/atkinson-pikettysaezJEL10.pdf

Arriagada Luco, C. \& Simioni, D. (2001). Dinámica de valorización del suelo en el área metropolitana del Gran Santiago y desafios del financiamiento urbano. Serie Medio Ambiente y Desarrollo n. ${ }^{\circ} 44$ (LC/L.1646-P). Santiago, Chile: CEpal. En http:// repositorio.cepal.org/bitstream/handle/11362/5738/S01111023_es.pdf?sequence=1

Bartels, L. M. (2009). Unequal democracy: The political economy of the new gilded age. Princeton, NJ: Princeton University Press.

Bell, W. (1954). A probability model for the measurement of ecological segregation. Social Forces, 32 (4), 357-364. doi: 10.2307/2574118

Borsdorf, A. (2003). Cómo modelar el desarrollo y la dinámica de la ciudad latinoamericana. EURE, 29 (86), 37-49. En http://dx.doi.org/10.4067/S0250-71612003008600002

Duncan, O. D. \& Duncan, B. (1955). A methodological analysis of segregation indexes. American Sociological Review, 20 (2), 210-217. doi: 10.2307/2088328

Elbers, C., Lanjouw, J. O. \& Lanjouw, P. (2003). Micro-level estimation of poverty and inequality. Econometrica, 71 (1), 355-364. doi: 10.1111/1468-0262.00399

Erbe, B. M. (1975). Race and socioeconomic segregation. American Sociological Review, 40 (6), 801-812.

Fossett, M. (2005). Measuring uneven distribution: conceptual and practical considerations for segregation studies. En Annual meeting of the American Sociological Association, Marriott Hotel, Loews Philadelphia Hotel, Philadelphia, PA, 12 agosto 2005.

Glaeser, E. L. \& Vigdor, J. L. (2003). Racial segregation: Promising news. En B. Katz \& R. E. Lang (Eds.), Redefining Urban \& Suburban America: Evidence from Census 2000 (Vol. 1, pp. 2011-234). Washington, D.c.: The Brookings Institute..

Hacker, J. S. \& Pierson, P. (2011). Winner-take-all politics: How Washington made the rich richer-and turned its back on the middle class. Nueva York: Simon and Schuster. 
Hentschel, J., Lanjouw, J., Lanjouw, P. \& Poggi, J. (1999). Combining survey data with census data to construct spatially disaggregated poverty maps: A case study of Ecuador. World Economic Bank Review, 14 (1), 147-166.

Iceland, J. \& Wilkes, R. (2006). Does socioeconomic status matter? Race, class, and residential segregation. Social Problems, 53 (2), 248-273. doi: 10.1525/sp.2006.53.2.248

Lambiri, D. \& Vargas, M. (2011). Residential segregation and public housing policy, the case of Chile. Documento de Trabajo n. ${ }^{\circ}$ 29, Facultad de Economía y Empresa, Universidad Diego Portales, Chile. En http://bit.ly/207dVSG

Massey, D. S. \& Denton, N. A. (1988). The dimensions of residential segregation. Social Forces, 67 (2), 281-315. doi: 10.1093/sf/67.2.281

Monkkonen, P. (2012). La segregación residencial en el México urbano. EURE, 38 (114), 125146. En http://www.scielo.cl/pdf/eure/v38n114/art05.pdf

Opazo, M. (2014). Evaluación de la política de vivienda social en la década de los noventa sobre la segregación y movilidad residencial en Chile. Tesis para la obtención del grado de doctor, Universidad Autónoma de Madrid. En https://dialnet.unirioja.es/servlet/ tesis? codigo $=43761$

Organisation for Economic Co-operation and Development (OECD). (2010). Pisa 2009 Results: Overcoming social background. Equity in learning opportunities and outcomes (Volume II). En http://dx.doi.org/10.1787/9789264091504-en (https://www.oecd. org/pisa/pisaproducts/48852584.pdf)

Piketty, T. (2014), Capital in the XXI Century. Cambridge, MA: The Belknap Press of Harvard University Press.

Poduje, I. (2011). Estudio "Guetos en Chile" [En línea]. ATISBA, Estudios \& Proyectos Urbanos. En http://www.docfoc.com/guetos-en-chile-atisba-monitor-pdf

Reardon, S. F. (2009). Measures of ordinal segregation. En Y. Flückiger, S. F. Reardon \& J. Silber (Eds.), Occupational and residential segregation (Research on Economic Inequality). (Vol. 17, pp. 129-155). Bingley, uk: Emerald. doi: 10.1108/S10492585(2009)0000017011

Reardon, S. F. \& Bischoff, K. (2011). Income inequality and income segregation. American Journal of Sociology, 116 (4), 1092-1153. doi: 10.1086/657114

Reardon, S. F. \& O'Sullivan, D. (2004). Measures of spatial segregation. Sociological Methodology, 34 (1), 121-162. doi: 10.1111/j.0081-1750.2004.00150.x

Rodríguez, J. (2001). Segregación residencial socioeconómica: ¿qué es?, ¿cómo se mide?, ¿qué está pasando?, ¿importa? Serie Población y Desarrollo, n. 16 (LC/L.1576-P). Santiago, Chile: CEPAL. En http://repositorio.cepal.org/bitstream/handle/11362/7149/ S017595_es.pdf?sequence $=1$

Rodríguez, J. \& Arriagada, C. (2004). Segregación residencial en la ciudad latinoamericana. EURE, 30 (89), 5-24. En http://dx.doi.org/10.4067/S0250-71612004008900001

Ruiz-Tagle, J. \& López, E. (2014). El estudio de la segregación residencial en Santiago de Chile: revisión crítica de algunos problemas metodológicos y conceptuales. EURE, 40 (119), 25-48. http://dx.doi.org/10.4067/S0250-71612014000100002

Sabatini, F. (2006). La segregación social del espacio en las ciudades de América Latina. Washington, D.C.: Banco Interamericano de Desarrollo. En https://publications.iadb. org/handle/11319/5324 
Sabatini, F. \& Brain, I. (2008). La segregación, los guetos y la integración social urbana: mitos y claves. EURE, 34 (103), 5-26. En http://dx.doi.org/10.4067/S025071612008000300001

Sabatini, F., Cáceres, G. \& Cerda, J. (2001). Segregación residencial en las principales ciudades chilenas: Tendencias de las tres últimas décadas y posibles cursos de acción. EURE, 27 (82), 21-42. En http://dx.doi.org/10.4067/S0250-71612001008200002

Sabatini, F., Wormald, G., Sierralta, C. \& Peters, P. (2010). Segregación residencial en Santiago: Tendencias 1992-2002 y efectos vinculados con su escala geográfica. En F. Sabatini, R. Salcedo, G. Wormald \& G. Cáceres (Eds.), Tendencias de la segregación en las principales ciudades chilenas: Análisis censal 1982-2002 (pp. 19-42). Santiago: Pontificia Universidad Católica de Chile/Instituto Nacional de Estadísticas.

Sanhueza, C. \& Larrañaga, O. (2007). Residential segregation effects on poor's opportunities in Chile. Documentos de Trabajo n. ${ }^{\circ} 259$, Departamento de Economía, Universidad de Chile.

Telles, E. E. (1995). Structural sources of socioeconomic segregation in Brazilian metropolitan areas. The American Journal of Sociology, 100 (5), 1199-1223.

Watson, T. (2009). Inequality and the measurement of residential segregation by income in American neighborhoods. Review of Income and Wealth, 55 (3), 820-844. doi: $10.1111 /$ j.1475-4991.2009.00346.x

Wilkes, R. \& Iceland, J. (2004). Hypersegregation in the twenty-first century. Demography 41 (1), 23-36. En http://link.springer.com/article/10.1353\%2Fdem.2004.0009\#page-1 


\section{ANEXO}

CUADRo AI | Población y contribución comunal al CGI, Gran Santiago 1992 y 2002

\begin{tabular}{|c|c|c|c|c|c|c|c|}
\hline COMUNA & $\begin{array}{c}\text { POBLACIÓN } \\
1992\end{array}$ & $\mid \begin{array}{c}\text { POBLACIÓN } \\
2002\end{array}$ & $\begin{array}{c}\text { CONTRIBUCIÓN } \\
\text { COMUNAL } \\
\text { CGI I992 }\end{array}$ & \begin{tabular}{|c} 
DESV. STD. \\
1992 \\
$\mathrm{~N}=\mathrm{IOO}$
\end{tabular} & $\begin{array}{c}\text { CONTRIBUCIÓN } \\
\text { COMUNAL } \\
\text { CGI } 2002\end{array}$ & $\begin{array}{c}\text { DESV. } \\
\text { STD. } \\
2002 \\
\mathrm{~N}=100\end{array}$ & $\begin{array}{c}\text { САMBIO } \\
\text { CONTRIBUCIÓN } \\
\text { I992-2002 }\end{array}$ \\
\hline Santiago & 215.170 & 184.709 & 0,132 & 0,0289 & 0,170 & 0,0254 & $0,0382^{*}$ \\
\hline Cerrillos & 71.237 & 71.105 & 0,163 & 0,0123 & 0,169 & 0,0111 & 0,0057 \\
\hline Cerro Navia & 155.641 & 148.100 & 0,181 & 0,0212 & 0,179 & 0,0269 & $-0,0017$ \\
\hline Conchalí & 152.616 & 132.823 & 0,148 & 0,0150 & 0,158 & 0,0108 & $0,0100^{*}$ \\
\hline El Bosque & 172.145 & 173.694 & 0,171 & 0,0170 & 0,175 & 0,0220 & 0,0040 \\
\hline Estación Central & 137.761 & 123.939 & 0,140 & 0,0152 & 0,154 & 0,0083 & $0,0135^{*}$ \\
\hline Huechuraba & 61.735 & 73.965 & 0,180 & 0,0253 & 0,215 & 0,0173 & $0,0353^{*}$ \\
\hline Independencia & 75.725 & 63.078 & 0,123 & 0,0280 & 0,127 & 0,0115 & 0,0044 \\
\hline La Cisterna & 93.395 & 84.187 & 0,116 & 0,0157 & 0,113 & 0,0094 & $-0,0032^{*}$ \\
\hline La Florida & 328.802 & 364.070 & 0,155 & 0,0148 & 0,169 & 0,0078 & $0,0144^{*}$ \\
\hline La Granja & 132.857 & 132.154 & 0,163 & 0,0189 & 0,172 & 0,0227 & $0,0081^{*}$ \\
\hline La Pintana & 169.601 & 189.502 & 0,234 & 0,0350 & 0,215 & 0,0353 & $-0,0188^{*}$ \\
\hline La Reina & 91.360 & 95.575 & 0,337 & 0,0909 & 0,280 & 0,0326 & $-0,0570^{*}$ \\
\hline Las Condes & 205.367 & 246.128 & 0,385 & 0,0867 & 0,351 & 0,0499 & $-0,0342^{*}$ \\
\hline Lo Barnechea & 47.100 & 73.127 & 0,200 & 0,0330 & 0,277 & 0,0264 & $0,0772^{*}$ \\
\hline Lo Espejo & 120.054 & 112.500 & 0,177 & 0,0231 & 0,190 & 0,0232 & $0,0123^{*}$ \\
\hline Lo Prado & 110.731 & 104.116 & 0,155 & 0,0161 & 0,159 & 0,0131 & 0,0034 \\
\hline Macul & 119.644 & 111.000 & 0,151 & 0,0214 & 0,168 & 0,0149 & $0,0175^{*}$ \\
\hline Maipú & 256.078 & 466.440 & 0,169 & 0,0206 & 0,182 & 0,0088 & $0,0136^{*}$ \\
\hline Ńuñoa & 169.637 & 160.636 & 0,249 & 0,0692 & 0,255 & 0,0376 & 0,0063 \\
\hline P. Aguirre Cerda & 130.253 & 114.211 & 0,143 & 0,0167 & 0,157 & 0,0122 & $0,0144^{*}$ \\
\hline Peñalolén & 179.002 & 215.593 & 0,173 & 0,0195 & 0,200 & 0,0109 & $0,0274^{*}$ \\
\hline Providencia & 105.430 & 114.481 & 0,478 & 0,1587 & 0,331 & 0,0539 & $-0,1464^{*}$ \\
\hline Pudahuel & 137.213 & 195.005 & 0,180 & 0,0220 & 0,192 & 0,0186 & $0,0123^{*}$ \\
\hline Quilicura & 41.093 & 126.396 & 0,198 & 0,0188 & 0,212 & 0,0131 & $0,0144^{*}$ \\
\hline Quinta Normal & 115.602 & 103.051 & 0,107 & 0,0161 & 0,119 & 0,0117 & $0,0123^{*}$ \\
\hline Recoleta & 162.812 & 145.694 & 0,133 & 0,0165 & 0,145 & 0,0113 & $0,0115^{*}$ \\
\hline Renca & 128.470 & 133.263 & 0,181 & 0,0219 & 0,185 & 0,0268 & 0,0040 \\
\hline San Joaquín & 113.354 & 96.203 & 0,137 & 0,0206 & 0,153 & 0,0105 & $0,0168^{*}$ \\
\hline San Miguel & 79.235 & 75.222 & 0,136 & 0,0299 & 0,161 & 0,0159 & $0,0247^{*}$ \\
\hline San Ramón & 100.775 & 94.446 & 0,169 & 0,0245 & 0,175 & 0,0244 & 0,0054 \\
\hline Vitacura & 78.661 & 80.558 & 0,518 & 0,1353 & 0,400 & 0,0437 & $-0,1184^{*}$ \\
\hline Puente Alto & 250.703 & 488.796 & 0,176 & 0,0127 & 0,196 & 0,0128 & $0,0201^{*}$ \\
\hline San Bernardo & 187.985 & 244.564 & 0,178 & 0,0176 & 0,193 & 0,0164 & $0,0145^{*}$ \\
\hline Gran Santiago & 4.697 .244 & 5.338 .331 & 0,192 & 0,0171 & 0,199 & 0,0103 & $0,0074^{*}$ \\
\hline
\end{tabular}

NOTAS (I) LOS DATOS DE POBLACIÓN SON DE LOS CENSOS I 992 Y 2002. LOS ÍNDICES Y DESVIACIONES ESTÁNDAR SON DE ELABORACIÓN PROPIA. (2) $\left(^{*}\right)$ EN LA ÚLTIMA COLUMNA SE INDICA UN CAMBIO EN LA CONTRIBUCIÓN ENTRE I 992 Y 2002 ESTADÍSTICAMENTE SIGNIFICATIVO (P<O.OI). (3) LA DESVIACIÓN ESTÁNDAR EN CADA AÑO SE CALCULA A PARTIR DE IOO ITERACIONES DEL CÁLCULO DE INGRESO POR HOGAR.

FUENTE ELABORACIÓN PROPIA 